\title{
Risk Factors for the Development of Cataract Requiring Surgery in Uveitis Associated with Juvenile Idiopathic Arthritis
}

\author{
KAREN M. SIJSSENS, ANIKI ROTHOVA, DAVID A. M. C. VAN DE VIJVER, JAN S. STILMA, \\ AND JOKE H. DE BOER
}

- PURPOSE: To identify the possible risk factors for the development of cataract requiring surgery in children with juvenile idiopathic arthritis (JIA)-associated uveitis.

- DESIGN: Retrospective cohort study.

- METHODS: Data of 53 children with JIA-associated uveitis, of whom 27 had undergone cataract extraction (CE), were obtained. The main outcome measure, the interval between the onset of uveitis and the first $\mathrm{CE}$ (U-CE interval), was examined in relation to clinical and ophthalmologic characteristics and treatment strategies before CE.

- RESUlTs: A shorter U-CE interval was found for children with posterior synechia vs those without posterior synechia (hazard ratio $[\mathrm{HR}], 3.57$; 95\% confidence interval [CI], 1.33 to 10.00 ). No significant difference was found for children in whom the uveitis was the first manifestation of JIA vs those in whom arthritis was the first manifestation of JIA ( HR, 1.59; 95\% CI, 0.63 to 4.00) and children treated with periocular corticosteroid injections vs those not treated with periocular corticosteroid injections (HR, 3.23; 95\% CI, 0.95 to 11.11 ). Children treated with methotrexate (MTX) had a longer U-CE interval than children not treated with MTX (HR, 0.29 ; $95 \% \mathrm{CI}, 0.10$ to 0.87 ).

- CONCLUSIONS: The risk factor for development of early cataract requiring surgery in children with JIAassociated uveitis is the presence of posterior synechia at the time of diagnosis of uveitis. However, early treatment with MTX is associated with a mean delay in the development of cataract requiring surgery of 3.5 years. (Am J Ophthalmol 2007;144:574-579. (C) 2007 by Elsevier Inc. All rights reserved.)

Accepted for publication Jun 19, 2007.

From the FC Donders Institute of Ophthalmology, University Medical Center Utrecht, Utrecht, The Netherlands (K.M.S., A.R., J.S.S., J.H.d.B.); and the Department of Virology, Eijkman Winkler Center, University Medical Center Utrecht, Utrecht, The Netherlands (D.A.M.C.v.d.V.).

Inquiries to Karen M. Sijssens, FC Donders Institute of Ophthalmology, University Medical Center Utrecht, E.03.136, Heidelberglaan 100, 3584 CX Utrecht, The Netherlands; e-mail: K.Sijssens@umcutrecht.nl

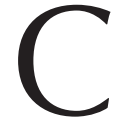
ATARACT REPRESENTS A MAJOR COMPLICATION of uveitis in childhood. It occurs in approximately $35 \%$ (range, $20 \%$ to $70 \%$ ) of the cases of juvenile idiopathic arthritis (JIA)-associated uveitis. ${ }^{1}$ Cataract can be caused by systemic and local corticosteroid treatment and the intraocular inflammation itself. ${ }^{2}$ The risk of cataract formation in JIA-associated uveitis increases when posterior synechia are present at the initial examination and with treatment with a high dose of systemic corticosteroids. ${ }^{3}$ Previously, the visual outcome of cataract surgery in JIA-associated uveitis was poor. Since new surgical techniques have been recommended, the results of cataract extraction (CE) have improved, but surgery in uveitic eyes of children still remains challenging. 4

The aim of our study was to evaluate which factors accelerate the development of cataract requiring surgery in JIA-associated uveitis. Therefore, clinical and ophthalmologic characteristics and the treatment strategies in children with JIA-associated uveitis were investigated in relation to the interval between the diagnosis of uveitis and the first cataract extraction (U-CE interval).

\section{METHODS}

WE REVIEWED THE MEDICAL RECORDS OF 53 CHILDREN with JIA-associated uveitis $(n=51)$ or antinuclear antibody (ANA)-positive uveitis without arthritis $(n=2)$ diagnosed before the age of 16 years. These children represent all children with JIA-associated uveitis identified in a complete database search of the FC Donders Institute of Ophthalmology, University Medical Center, Utrecht, The Netherlands, from January 1990 through June 2006 (53 of 240 children with uveitis). Our center combines a secondary and a tertiary referral function. Children were referred by the ophthalmologists of secondary referral hospitals or by the pediatric rheumatologists of our medical center. The pediatric rheumatologists referred all children with JIA for uveitis screening according to the criteria of the American Academy of Pediatrics in cases of JIA. 5 The parents of the children gave permission for all the treatment.

In this study, we investigated the clinical data of the first eye that underwent cataract surgery per patient. Cataract 
TABLE 1. The First Manifestation of Juvenile Idiopathic Arthritis, the Presence of Posterior Synechia, and the Treatment with Methotrexate and Periocular Corticosteroid Injection(s) in Children with Uveitis Associated with Juvenile Idiopathic Arthritis in Relation to Clinical Characteristics and Treatment Strategies

\begin{tabular}{|c|c|c|c|c|c|c|c|c|c|c|c|c|}
\hline \multirow[b]{3}{*}{ Clinical Characteristics } & \multicolumn{12}{|c|}{ Children with Uveitis Associated with JIA $(n=53)$} \\
\hline & \multicolumn{3}{|c|}{ First Manifestation of JIA ( $n=53)$} & \multicolumn{3}{|c|}{$\begin{array}{l}\text { Posterior Synechia at the Time of } \\
\text { Diagnosis of Uveitis }\left(n=48^{*}\right)\end{array}$} & \multicolumn{3}{|c|}{ Treatment with MTX $\left(n=42^{\dagger}\right)$} & \multicolumn{3}{|c|}{$\begin{array}{l}\text { Treatment with Periocular Corticosteroid } \\
\text { Injection(s) }\left(n=49^{\ddagger}\right)\end{array}$} \\
\hline & Uveitis $(n=12)$ & Arthritis $(n=41)$ & $P$ value & Yes $(n=15)$ & No $(n=33)$ & $P$ value & $\begin{array}{l}\text { During the First Year } \\
\text { after the Diagnosis } \\
\text { of Uveitis }(n=17)\end{array}$ & $\begin{array}{l}\text { Never Treated with MTX } \\
\qquad(\mathrm{n}=25)\end{array}$ & $P$ value & $\begin{array}{l}\text { During the First Year } \\
\text { after the Diagnosis } \\
\text { of Uveitis }(n=7)\end{array}$ & Never $(n=42)$ & $P$ value \\
\hline \multicolumn{13}{|c|}{ Mean age (yrs) at diagnosis of } \\
\hline uveitis (median) & $4.7(4.3)$ & $5.6(4.8)$ & .750 & $5.3(4.5)$ & $5.5(5.0)$ & .956 & $6.3(3.4)$ & $5.3(4.8)$ & .431 & $5.0(4.1)$ & $5.7(4.9)$ & .547 \\
\hline Female-to-male ratio & $5: 7$ & $30: 11$ & .080 & $8: 7$ & $23: 10$ & .276 & $12: 5$ & $16: 9$ & .747 & $3: 4$ & $29: 13$ & .217 \\
\hline \multicolumn{13}{|l|}{ Uveitis as the initial } \\
\hline manifestation of JIA & N.A. & N.A. & N.A. & $7 / 15$ & $4 / 33$ & .022 & $0 / 17$ & $10 / 25$ & .003 & $3 / 7$ & $9 / 42$ & .340 \\
\hline ANA positive ${ }^{\S}$ & $10 / 12$ & $35 / 40$ & .656 & $12 / 15$ & $30 / 33$ & .360 & $15 / 17$ & $22 / 24$ & 1.000 & $7 / 7$ & $34 / 41$ & .573 \\
\hline HLA-B27 positive ${ }^{\S}$ & $1 / 8$ & $2 / 11$ & 1.000 & $0 / 8$ & $3 / 9$ & .206 & $1 / 3$ & $0 / 10$ & .231 & $0 / 1$ & $3 / 18$ & 1.000 \\
\hline \multicolumn{13}{|l|}{ Presence of posterior } \\
\hline \multicolumn{13}{|l|}{ synechia at time of } \\
\hline diagnosis of uveitis $\|$ & $7 / 11$ & $8 / 37$ & .022 & N.A. & N.A. & N.A. & $4 / 17$ & $9 / 21$ & .207 & $5 / 6$ & $10 / 38$ & .013 \\
\hline \multicolumn{13}{|l|}{ Lens opacity at time of } \\
\hline diagnosis of uveitis" & $1 / 9$ & $2 / 36$ & .497 & $1 / 5$ & $2 / 36$ & .330 & $1 / 17$ & $2 / 18$ & 1.000 & $1 / 5$ & $2 / 36$ & .330 \\
\hline \multicolumn{13}{|l|}{ Treatment with MTX during } \\
\hline \multicolumn{13}{|l|}{ the first year after the } \\
\hline diagnosis of uveitis & $0 / 12$ & $17 / 41$ & .005 & $4 / 15$ & $13 / 33$ & .387 & N.A. & N.A. & N.A. & $2 / 7$ & $13 / 42$ & 1.000 \\
\hline \multicolumn{13}{|l|}{ Treatment with periocular } \\
\hline \multicolumn{13}{|l|}{ corticosteroid injection(s) } \\
\hline \multicolumn{13}{|l|}{ during the first year after } \\
\hline the diagnosis of uveitis & $3 / 12$ & $4 / 41$ & .183 & $5 / 15$ & $1 / 33$ & .008 & $2 / 17$ & $5 / 25$ & .681 & N.A. & N.A. & N.A. \\
\hline \multicolumn{13}{|l|}{ Treatment with systemic } \\
\hline \multicolumn{13}{|c|}{ corticosteroids during the } \\
\hline \multicolumn{13}{|c|}{ first year after the diagnosis } \\
\hline of uveitis & $0 / 12$ & $1 / 41$ & 1.000 & $1 / 15$ & $0 / 33$ & .313 & $1 / 17$ & $0 / 25$ & .405 & $1 / 7$ & $0 / 42$ & .143 \\
\hline \multicolumn{13}{|c|}{ Glaucoma surgery before CS } \\
\hline or the end of follow-up & $1 / 12$ & $8 / 41$ & .665 & $1 / 15$ & $8 / 33$ & .239 & $3 / 17$ & $1 / 25$ & .286 & $1 / 7$ & $5 / 42$ & 1.000 \\
\hline $\begin{array}{l}\text { ANA = antinuclear anti } \\
{ }^{*} \text { For five patients, no in } \\
{ }^{\dagger} \text { Eleven patients were t } \\
{ }^{\text {}} \text { Four patients were tre } \\
\text { §The ANA and HLA-B2 } \\
\text { "Data about posterior s }\end{array}$ & $\begin{array}{l}\text { CS = cataract } \\
\text { tion about post } \\
\text { with methotre } \\
\text { vith periocular } \\
\text { is were not ave } \\
\text { ia and lens ope }\end{array}$ & $\begin{array}{l}\text { surgery; HLA = } \\
\text { terior synechia } \\
\text { xate more than } \\
\text { corticosteroid in } \\
\text { ailable for all ch } \\
\text { acity at diagnos }\end{array}$ & $\begin{array}{l}=\text { humar } \\
\text { at time c } \\
\text { one yea } \\
\text { jection( } \\
\text { ildren. } \\
\text { is of uve }\end{array}$ & $\begin{array}{l}\text { leukocyte ar } \\
\text { f diagnosis o } \\
\text { ir after the or } \\
\text { ) more than } \\
\text { itis was not }\end{array}$ & $\begin{array}{l}\text { ntigen; JIA } \\
\text { of uveitis wa } \\
\text { nset of uveit } \\
\text { one year af } \\
\text { available for }\end{array}$ & $\begin{array}{l}=\text { juveni } \\
\text { as availa } \\
\text { tis. } \\
\text { ter the o } \\
\text { all chilc }\end{array}$ & $\begin{array}{l}\text { le idiopathic arthrit } \\
\text { ble. } \\
\text { onset of uveitis. } \\
\text { dren. }\end{array}$ & is; $M T X=$ methotrex & ate; N.A. & = not applicable. & & \\
\hline
\end{tabular}


TABLE 2. Hazard Ratios (Crude and Adjusted) for Cataract Requiring Surgery in Children with Uveitis Associated with Juvenile Idiopathic Arthritis

\begin{tabular}{|c|c|c|c|}
\hline Variable & Crude HR (95\% Cl) & Adjustment for & Adjusted HR (95\% Cl) \\
\hline $\begin{array}{l}\text { Uveitis as the initial manifestation of juvenile } \\
\text { idiopathic arthritis (uveitis vs arthritis) }\end{array}$ & $2.44(1.09$ to 5.26$)$ & $\begin{array}{l}\text { The presence of posterior synechia at time of } \\
\text { diagnosis of uveitis }\end{array}$ & 1.59 (0.63 to 4.00$)$ \\
\hline Gender (boys vs girls) & $1.64(0.71$ to 3.85$)$ & & \\
\hline ANA status (positive vs negative) & 0.88 (0.30 to 2.63$)$ & & \\
\hline Posterior synechia at time of diagnosis of & 4.55 (1.82 to 11.11$)$ & Treatment with periocular corticosteroid injection(s) & 3.57 (1.33 to 10.00$)$ \\
\hline \multirow[t]{2}{*}{ uveitis (yes vs no) } & & during the first year after the diagnosis of uveitis & \\
\hline & & and the course of juvenile idiopathic arthritis & \\
\hline Systemic treatment with methotrexate & $0.29(0.10$ to 0.87$)$ & & \\
\hline \multicolumn{4}{|l|}{ during the first year after the diagnosis of } \\
\hline uveitis (yes vs never) & & & \\
\hline Treatment with periocular corticosteroid & $5.26(1.89$ to 14.29$)$ & The presence of posterior synechia at time of & $3.23(0.95$ to 11.11$)$ \\
\hline injection(s) during the first year after the & & diagnosis of uveitis & \\
\hline diagnosis of uveitis (yes vs never) & & & \\
\hline $\begin{array}{l}\text { ANA = antinuclear antibody } ; \mathrm{Cl}=\text { confider } \\
\text { The HR was defined to be significant if on }\end{array}$ & $\begin{array}{l}\text { nce interval; } \mathrm{HR}=\text { ha } \\
\text { e did not fall into the }\end{array}$ & $\begin{array}{l}\text { azard ratio. } \\
95 \% \text { confidence interval. }\end{array}$ & \\
\hline
\end{tabular}

surgery to the second eye was not included in this study because possible personal predispositions may interfere with the results. If no CE was performed before the end of follow-up, the data of the first affected eye was included. We recorded the following clinical and ophthalmologic data for each patient: gender, age at diagnosis of uveitis and arthritis, the course of JIA (arthritis or uveitis as the first manifestation of JIA), ANA status, adherent posterior synechia and lens opacity at the time of the diagnosis of uveitis, age at first cataract surgery, all intraocular surgeries before $\mathrm{CE}$, and age at last visit to our clinic. Furthermore, we noted all treatment in the first year after the diagnosis of uveitis and before CE or final visit and paid special interest to treatment with methotrexate (MTX), systemic corticosteroids, and periocular corticosteroids. We compared children with the above-mentioned treatment strategies during the first year after the onset of uveitis with children who had never been treated with those drugs. Treatment during the first year was chosen because after more than one year, cataract formation might have developed as a result of other factors. The treatment with corticosteroid drops was not specifically investigated because this treatment method was used in all children.

All intraocular surgeries before CE $(\mathrm{n}=9)$ were registered, and all were found to be glaucoma related (trabeculectomy with or without mitomycin C). Cataract surgery was performed if the visual acuity was 20/63 (Snellen) or less. The U-CE interval in the first operated eye was taken as the outcome parameter time until development of cataract requiring surgery. All patients who underwent CE were seen for follow-up. In this study, the follow-up period was defined as the time period between the diagnosis of uveitis and the last ocular examination or CE. The mean follow-up was 3.4 years (range, 0.2 to 14.1 years). Uveitis was classified and categorized according to the criteria of the Standardization of Uveitis Nomenclature Working Group. ${ }^{6}$ The diagnosis of JIA was made according to the criteria from the International League against Rheumatism. ${ }^{7,8}$ In cases of presumed JIA, the diagnosis was confirmed by a pediatric rheumatologist.

Statistical analysis of the data was performed by using the SPSS statistical software package version 12.0.1 (SPSS, Inc, Chicago, Illinois, USA). The interquartile range (IQR) was used to show the range between the first and third quartile. The Chi-square test or the Fisher exact test were used to compare categorical data. The independent samples $t$ test was used to compare the means of two groups. The mean U-CE interval was calculated with the Kaplan-Meier survival analysis, which corrects for patients who did not undergo CE before the end of follow-up. The Kaplan-Meier survival analyses were quantified using multivariate Cox proportional hazard analysis (i.e., time-toevent analysis) in which we submitted all variables with a $P<.05$ in univariate analysis. ${ }^{9}$ The hazard ratio $(\mathrm{HR})$ was defined to be significant if one did not fall into the $95 \%$ confidence interval.

\section{RESULTS}

- GENERAl CHARACTERISTICS: Of all the children investigated in this study, 27 of $53(51 \%)$ underwent CE before the end of follow-up. The mean follow-up of children with and without CE was 3.8 years (IQR, 1.2 to 5.0 years; $\mathrm{n}=27)$ and 3.1 years (IQR, 1.0 to 4.1 years; $\mathrm{n}=$ $26)$, respectively $(P=.593)$. The mean age at onset of uveitis of children with and without cataract surgery was 4.6 years (IQR, 3.4 to 5.1 years) and 6.2 years (IQR, 3.2 to 8.5 years $)$, respectively $(P=.088)$. The uveitis was chronic 
and bilateral in all cases, except for two girls who had chronic unilateral uveitis. Data concerning the first manifestation of JIA, the presence of posterior synechia, and the treatment with MTX and periocular corticosteroid injection(s) in relation to clinical characteristics and treatment strategies are shown in Table 1.

- ClinicAl CHARACTERISTICS IN RELATION TO CATARACT REQUIRING SURGERY: Children in whom the diagnosis of uveitis was the initial manifestation of JIA (CE, 11/12 [92\%]) had a significantly shorter mean U-CE interval than children in whom arthritis preceded uveitis (3.5 years vs CE, 16/41 [39\%]; 6.6 years, respectively; Table 2). In the group in which uveitis was the first manifestation of JIA, there were no children treated with MTX in the first year after the diagnosis of uveitis (Table 1). Therefore, adjustment was performed only for the presence of posterior synechia at the time of diagnosis of uveitis. After adjustment, the difference between arthritis and uveitis as the initial manifestation of JIA did not reach significance (Table 2). If we limit our data to only those patients who had arthritis as the initial manifestation of JIA ( $\mathrm{n}=41)$, no statistically significant difference was found for the U-CE interval between children treated with MTX during the first year after the diagnosis of uveitis $(\mathrm{n}=17)$ and children never treated with MTX $(\mathrm{n}=15$; HR, 0.46; 95\% confidence interval [CI], 0.13 to 1.61 ).

In addition to the course of JIA, we also examined the gender and ANA status of the patients. No statistically significant difference was found for the mean U-CE interval between boys (CE, 11/18 [61\%]) and girls (CE, 16/35 [46\%]; 4.9 and 5.8 years, respectively; HR, 0.61; 95\% CI, 0.26 to 1.41), nor between ANA-positive children (CE, 22/45 [49\%]) and ANA-negative children (CE, 4/7 [57\%]; 6.2 and 5.9 years, respectively; HR, 1.13; 95\% CI, 0.38 to 3.34; Table 2). In one additional child with cataract, the ANA status was unknown.

\section{- OPHTHALMOLOGIC CHARACTERISTICS IN RELATION} TO CATARACT REQUIRING SURGERY: The presence of adherent posterior synechia at the time of diagnosis of uveitis (CE, 12/15 [80\%]) resulted in a significantly shorter mean U-CE interval than when no posterior synechia were present (CE, 10/33 [30\%]; 3.0 vs 8.5 years, respectively; Table 2). After adjustment for treatment with periocular corticosteroid injections in the first year after the diagnosis of uveitis and for the course of uveitis, the difference between the two groups remained significant (Tables 1 and 2). In five children, no information about posterior synechia at time of diagnosis of uveitis was available.

Three children were observed with lens opacity at the time of diagnosis of uveitis. Because of this small number, the relation between lens opacity and the development of cataract requiring surgery was not evaluated.
Children with CE who previously had undergone glaucoma surgery $(\mathrm{n}=7)$ and children without previous glaucoma surgery $(\mathrm{n}=20)$ had a mean U-CE interval of 5.4 and 3.2 years, respectively. The mean period between the diagnosis of uveitis and glaucoma surgery was 4.2 years, and the mean period between glaucoma surgery and CE was 0.8 years. Between these two groups, no significant differences were found concerning treatment in the first year after the diagnosis of uveitis with MTX and periocular corticosteroid injections $(P=1.000$ and $P=1.000$, respectively).

- SYSTEMIC TREATMENT WITH MTX: Children who started MTX treatment during the first year after the diagnosis of uveitis (CE, 5/17 [29\%]) had a significantly longer mean U-CE interval than children who were never treated with MTX (CE, 16/25 [64\%]; 7.0 vs 3.5 years, respectively; Table 2). In 11 patients, MTX was started more than one year after the onset of uveitis. The indication for starting MTX treatment was uveitis not responding to topical corticosteroid treatment in five children and arthritis in 12 children. In the group treated with MTX during the first year after the diagnosis of uveitis, significantly fewer children had uveitis as the initial manifestation of JIA than children never treated with MTX ( $P=.003$; Table 1$)$. Adjustment could not be performed for the course of uveitis, because there were no children with uveitis as the initial manifestation of JIA in the MTX-treated group. If we limited our data to only those patients who were never treated with MTX ( $\mathrm{n}=$ 25), then children with uveitis as the initial manifestation of JIA (CE, 9/10 [90\%] after 1.8 years) had a significantly shorter U-CE interval than children who had arthritis as the first manifestation (CE, 7/15 [47\%] after 5.1 years) with an HR of 3.13 (95\% CI, 1.08 to 9.09).

- SYSTEMIC TREATMENT WITH CORTICOSTEROIDS: The relation between systemic corticosteroid treatment and the development of cataract could not be evaluated because there was only one child who had started systemic corticosteroid treatment during the first year after the diagnosis of uveitis.

- TREATMENT WITH PERIOCULAR CORTICOSTEROID INJECTIONS: Seven children were treated with periocular corticosteroid injection(s) in the first year after the diagnosis of uveitis. Of these, five children received one injection (including one child not operated on for cataract) and one child received five injections, and for one child, the number of injections in the first year after the diagnosis of uveitis was unclear. These children (CE, 6/7 [86\%]) had a significantly shorter U-CE interval than children never treated with periocular corticosteroid injections (CE, 17/42 [40\%]; 1.8 vs 7.1 years, respectively; Table 2). Adjusting for the presence of posterior synechia at the time of diagnosis of uveitis did not reach signifi- 
cance, but there was still a trend toward earlier cataract formation in children treated with corticosteroid injections (Tables 1 and 2). Four patients were treated with periocular corticosteroid injection(s) more than one year after the onset of uveitis.

\section{DISCUSSION}

THIS STUDY DEMONSTRATES THAT THE PRESENCE OF ADherent posterior synechia at the time of diagnosis of uveitis is strongly associated with the early development of cataract requiring surgery in JIA-associated uveitis and that treatment with MTX in the first year after the diagnosis of uveitis is associated with a delay in cataract surgery. Previous studies have demonstrated that the presence of posterior synechia at the time of diagnosis of uveitis is associated with a poor visual prognosis in JIA-associated uveitis. ${ }^{3}$ Because of the association with poor visual outcome, detection of uveitis before the formation of posterior synechia development is recommended. Therefore, screening of uveitis should occur shortly after the onset of arthritis. Chia and associates have advocated for more intensive screening for uveitis in the first year after the diagnosis of JIA in the hope of reducing the rate of complications. ${ }^{10}$ In concordance with Chia and associates, we believe that more intensive screening in the early phase of JIA may result in less frequent development of posterior synechia and therefore less early development of cataract. When uveitis is diagnosed, prompt, careful follow-up should ensue, especially in cases where posterior synechia already are present. However, in children in whom uveitis precedes arthritis, early detection of uveitis is not possible because these children have the intraocular inflammation without notice. Furthermore, one can speculate that the presence of posterior synechia may be an indication for early treatment with MTX because this drug was associated with a delay proceeding to cataract surgery. In this study, the presence of posterior synechia was equal in both groups treated with MTX in the first year and in the group never treated with MTX.

In addition to the presence of posterior synechia, treatment with periocular corticosteroid injections in the first year after the diagnosis of uveitis showed a trend, although not a significant one, toward a more rapid cataract requiring surgery development. In our series, we do not know the duration of uveitis before the diagnosis. Therefore, we cannot determine whether this is of influence on the early development of cataract requiring surgery. Whether the differences found for children with posterior synechia and children treated with periocular corticosteroid injections were caused by a more intense uveitis or whether this difference is removed after adjustment for the unknown duration of uveitis before diagnosis is unclear.

We could not investigate the effect of treatment with systemic corticosteroids in our series because there was only one child who had received this treatment in the first year. However, literature reports that systemic corticosteroids enhance cataract formation. ${ }^{3}$

The only modifiable factor that may be associated with a delay in the development of cataract requiring surgery was early treatment with MTX. If MTX treatment was started during the first year after the diagnosis of uveitis, the development of cataract was not accelerated, but rather was postponed by a mean of 3.5 years. Because cataract development in JIA-associated uveitis is caused by various factors, it is difficult to determine the specific importance of a single factor. Unfortunately, adjustment for the course of uveitis (first manifestation of JIA) was not possible, because there were no children with uveitis as the initial manifestation of JIA in the group treated with MTX. Referral bias cannot be excluded because our uveitis clinic is a combined secondary and tertiary center. However, patients with uveitis as well as arthritis as the initial manifestation of JIA were referred by their ophthalmologist. A possible explanation for the lack of children with uveitis as the initial manifestation of JIA may be that presently, MTX is not (yet) administered as the first treatment of choice for uveitis when there are no signs of arthritis. Treatment with topical corticosteroids initially is indicated, and only severe cases are treated with MTX. So if the cases who received MTX have a higher baseline risk of developing cataract requiring surgery, the benefit of MTX in delaying cataract surgery may have been reduced artificially, and the benefit may be even greater than what we observed. However, the delay in cataract surgery is still a remarkable observation, because the other treatment strategies were associated with a shortening of the U-CE interval. A delay in cataract formation is very favorable for several reasons. It is well known that $\mathrm{CE}$ in children is complicated because of anatomic and functional characteristics, which include the small globe size, increased tissue reactivity, lower scleral rigidity, changing axial length, and the risk of amblyopia. ${ }^{11,12}$ Furthermore, with a delay in cataract formation, it will be easier to treat secondary posterior capsule opacification, to measure axial length and keratometry, and to perform capsulotomies. If cataract development can be postponed, the visual prognosis may improve, especially in young children in whom the period in which management to prevent amblyopia is necessary will be shortened. The protective mechanism responsible for the delay of development of cataract requiring surgery in children treated with MTX may include better control of the intraocular inflammation with possibly fewer flare-ups during the course of the disease. Another explanation may be that treatment with MTX diminishes the need for treatment with topical or systemic corticosteroids. However, both hypotheses need further investigation.

A limitation of this study is the relatively small number of patients available for statistical analysis, especially of some subgroups. Because of these small numbers, no analysis could be carried out on lens opacity and systemic corticosteroids in relation to the development of cataract requiring surgery. Furthermore, no patients were included 
who had been treated with MTX and who had uveitis as the initial manifestation of JIA, so adjustment for the course of JIA could not be performed in this subgroup. Another limitation is the possible bias resulting from the time of cataract surgery. All children had a visual acuity of 20/63 (Snellen) or less before CE. However, the final decision to perform cataract surgery is not based solely on visual acuity, but on a combination of visual acuity, the risk of amblyopia, clinical findings, and the symptoms and preferences of patients and parents. So the different time schedules adopted for performing CE may well have influenced the results.

What is positive about this study is that it included only children with JIA-associated uveitis, which means it is based on a homogeneous population. Furthermore, all the children were seen in the same institute, and the different analyses were all performed on one relatively large study group with a relatively long median follow-up of 3.4 years. For further study, it may be interesting to compare early treatment (during the first year after the diagnosis of uveitis), late treatment (treatment after more than one year after the onset of uveitis), and no treatment with MTX, periocular corticosteroid injections, or both in a large patient series.

In conclusion, development of cataract requiring surgery in JIA-associated uveitis is caused by various factors. The presence of adherent posterior synechia at the time of diagnosis of uveitis is strongly associated with the early development of cataract requiring surgery in children with JIA-associated uveitis. This is in contrast with early treatment with MTX, which is associated with a delay in the development of cataract requiring surgery. So the beneficial effect of early treatment with MTX on the development of cataract requiring surgery may be expected in cases with posterior synechia. These observations are of value for ophthalmologists treating chronic severe uveitis in children with JIA.

THIS STUDY WAS SUPPORTED BY THE DR. F. P. FISCHER FOUNDATION, UTRECHT, THE NETHERLANDS (DR SIJSSENS), AND BY the ViroLab Project, which is sponsored by the European Union, Contract IST-027446 (Dr van de Vijver). The authors indicate no financial conflict of interest. Involved in design and conduct of study (K.M.S., A.R., J.H.d.B.); collection and management of the data (K.M.S., J.H.d.B.); analysis and interpretation of the data (K.M.S., A.R., D.A.M.C.v.d.V., J.S.S., J.H.d.B.); preparation of the manuscript (K.M.S., J.H.d.B.); review of the manuscript (A.R., D.A.M.C.v.d.V., J.S.S., J.H.d.B.); and approval of the manuscript (K.M.S., A.R., D.A.M.C.v.d.V., J.S.S., J.H.d.B.).

\section{REFERENCES}

1. Edelsten C, Lee V, Bentley CR, et al. An evaluation of baseline risk factors predicting severity in juvenile idiopathic arthritis associated uveitis and other chronic anterior uveitis in early childhood. Br J Ophthalmol 2002;86:51-56.

2. Foster CS, Fong LP, Singh G. Cataract surgery and intraocular lens implantation in patients with uveitis. Ophthalmology 1989;96:281-288.

3. Wolf MD, Lichter PR, Ragsdale CG. Prognostic factors in the uveitis of juvenile rheumatoid arthritis. Ophthalmology 1987;94:1242-1248.

4. Foster CS, Barrett F. Cataract development and cataract surgery in patients with juvenile rheumatoid arthritis-associated iridocyclitis. Ophthalmology 1993;100:809-817.

5. American Academy of Pediatrics Section on Rheumatology and Section on Ophthalmology. Guidelines for ophthalmologic examinations in children with juvenile rheumatoid arthritis. Pediatrics 1993;92:295-296.

6. Jabs DA, Nussenblatt RB, Rosenbaum JT. Standardization of uveitis nomenclature for reporting clinical data. Results of the First International Workshop. Am J Ophthalmol 2005; 140:509-516.

7. Berntson L, Fasth A, Andersson-Gare B, et al. Construct validity of ILAR and EULAR criteria in juvenile idiopathic arthritis: a population based incidence study from the Nordic countries. International League of Associations for Rheumatology. European League Against Rheumatism. J Rheumatol 2001;28:2737-2743.

8. Krumrey-Langkammerer M, Hafner R. Evaluation of the ILAR criteria for juvenile idiopathic arthritis. J Rheumatol 2001;28:2544-2547.

9. Hosmer D, Lemeshow S. Applied logistic regression. New York, New York: Wiley Interscience, 1989:1-307.

10. Chia A, Fraco, Lee V, et al. Factors related to severe uveitis at diagnosis in children with juvenile idiopathic arthritis in a screening program. Am J Ophthalmol 2003;135:757-762.

11. Malukiewicz-Wisniewska G, Kaluzny J, Lesiewska-Junk H, Eliks I. Intraocular lens implantation in children and youth. J Pediatr Ophthalmol Strabismus 1999;36:129-133.

12. Wilson ME, Bluestein EC, Wang XH. Current trends in the use of intraocular lenses in children. J Cataract Refract Surg 1994;20:579-583. 


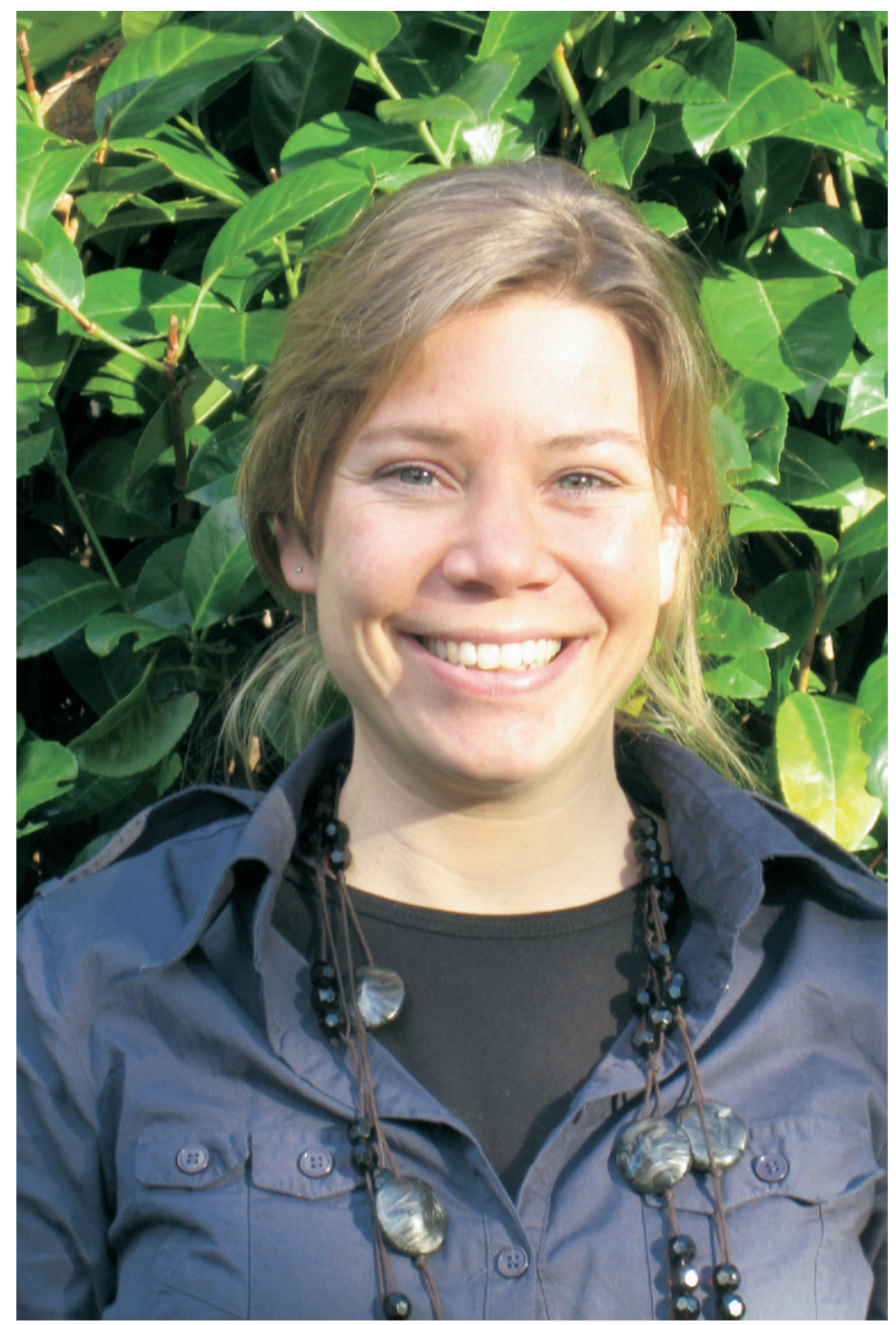

Biosketch

Karen M. Sijssens graduated in 2004 from the University Medical Center Utrecht, The Netherlands. Currently, she is pursuing her PhD degree about pediatric uveitis at the FC Donders Institute of Ophthalmology at the University Medical Center Utrecht, The Netherlands. Sijssens plans to begin her residency training in Ophthalmology in 2008. 